\title{
Characterization of Dislocation Plasticity in Rhenium using In-Situ TEM Deformation
}

\author{
Julian E.C. Sabisch ${ }^{1}$ and Andrew M. Minor ${ }^{1}$ \\ ${ }^{1 .}$ Department of Materials Science, University of California, Berkeley, USA, and the \\ National Center for Electron Microscopy, Molecular Foundry, Lawrence Berkeley National Laboratory, \\ USA
}

Rhenium is unusual among the refractory metals for having no ductile-to-brittle transition temperature as well as retaining much of its strength throughout the full range of operating temperatures [1,2]. Like all hexagonal close packed (HCP) metals, Re relies heavily on twinning in order to accommodate deformation, due to the lack of sufficient active slip planes for isotropic deformation. Previous work has shown the dominant twin mode in Re to be the $\{11-21\}<11-26>$ type twin, with the $\{11-22\}<11-23>$ and the $\{10-12\}<10-11>$ twin modes seen secondarily $[3,4,5]$. The active slip planes most seen during macroscopic deformation were $\{0001\}$ and $\{10-11\}$, with slip occurring secondarily on $\{10-11\}$ planes. While studies have been conducted using advanced electron microscopy techniques to better understand the interactions of twinning during deformation, the behavior of dislocations during deformation has yet to be fully characterized.

The goal of this work is to determine the fundamental deformation behavior of dislocations and twins in rhenium using both post mortem analysis and in-situ TEM techniques. The character of dislocations in rhenium is yet unknown, and underlying reasons for its excellent high temperature mechanical properties will be investigated using in-situ TEM tensile testing at high temperatures. As twinning has been shown to be the dominant deformation mechanism, any determination of unique twin-dislocation interactions in rhenium are important in order gain a full understanding of this unusual system.

In-situ straining was performed on a JOEL 3010 using a Gatan tensile heating-straining holder. Poststraining characterization of dislocations active during testing, seen in Figure 1, show that the dislocations are of screw type lying on the $\{10-10\}$ planes (determined from slip traces) with burgers vectors of $\langle 11-20\rangle$. Dislocations were shown to be active on all of the prism planes and readily able to accommodate the applied stain. Movies made of the dislocation motion show that in general the movement of dislocations is relatively "jerky", akin to prism slip in titanium. [6]

After the initial straining and determination of dislocation character in each sample, the samples were heated to $920{ }^{\circ} \mathrm{C}$ and strained further. The behavior of dislocations did not change with the increased temperature. As the melting temperature of rhenium is $3180{ }^{\circ} \mathrm{C}$, this elevated operating temperature does not quite reach one third of its melting temperature. The "jerky" motion of dislocations remained consistent at high temperatures.

It has been shown that dislocations in rhenium are predominantly $<$ a $>$ type $<11-20>$ dislocations acting on the prism planes, with the deformation behavior of rhenium being unaffected by temperatures up to $920{ }^{\circ} \mathrm{C}$. Further work will examine Re samples deformed ex-situ to different amounts of strain and characterized using TEM and EBSD to determine the relative behavior of dislocation plasticity and twinning in this interesting system [7]. 


\section{References:}

[1] B. D. Bryskin, Proceedings of the Ninth Symposium on Space Nuclear Power Systems. 246 (1992), p. 278.

[2] B. D. Bryskin, F. C, Danek, JOM 43-7 (1991) p. 24.

[3] A. Chruchman, Transactions of the American Institue of Mining and Metallurgical Engineers 218-2 (1960) p.262.

[4] J. Kacher, A. M. Minor, Acta Materialia 81 (2014) p.1.

[5] K. Peter et al,Twinning in Advanced Materials 1 (1993) p.475.

[6] E. Clouet et al, Nature Materials. 14 (2015) p. 931.

[7] We gratefully acknowledge funding from the US Office of Naval Research under Grant No. N00014-11-1-0886. Work at the Molecular Foundry at Lawrence Berkeley National Laboratory was supported by the U.S. Department of Energy under Contract \# DE-AC02-05CH11231.
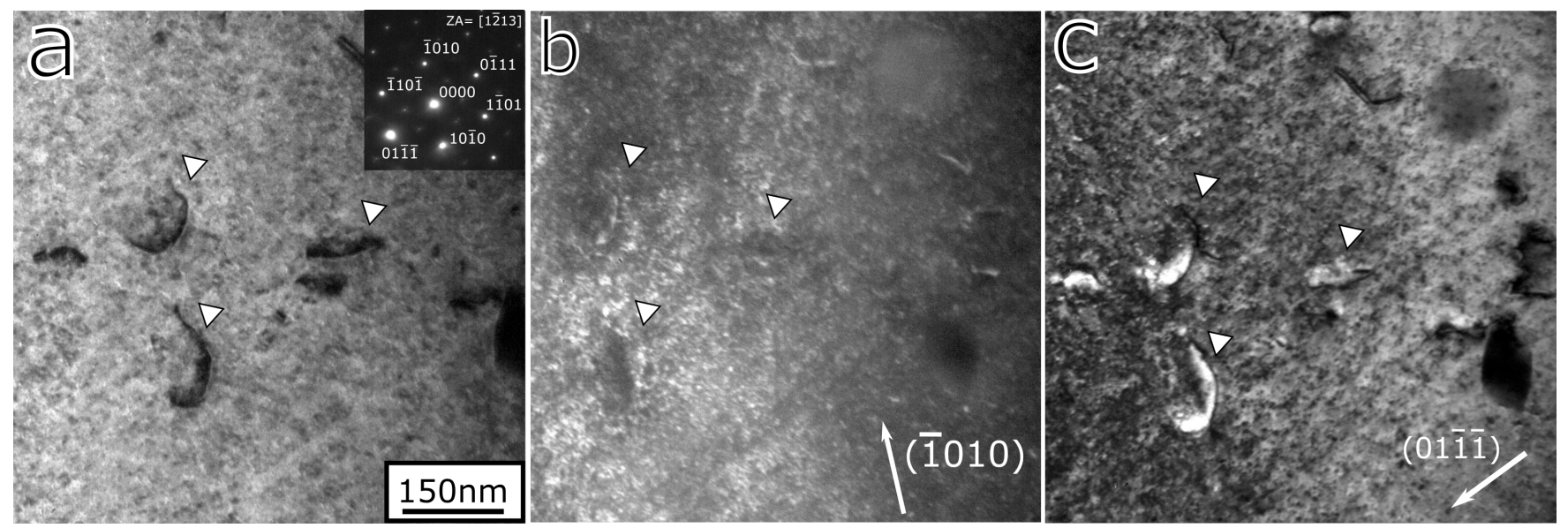

Figure 1. Dislocation analysis from active region of in-situ sample. a) Brightfield image of dislocations marked with triangles. b,c) Different darkfield conditions which show visible and invisible dislocations.
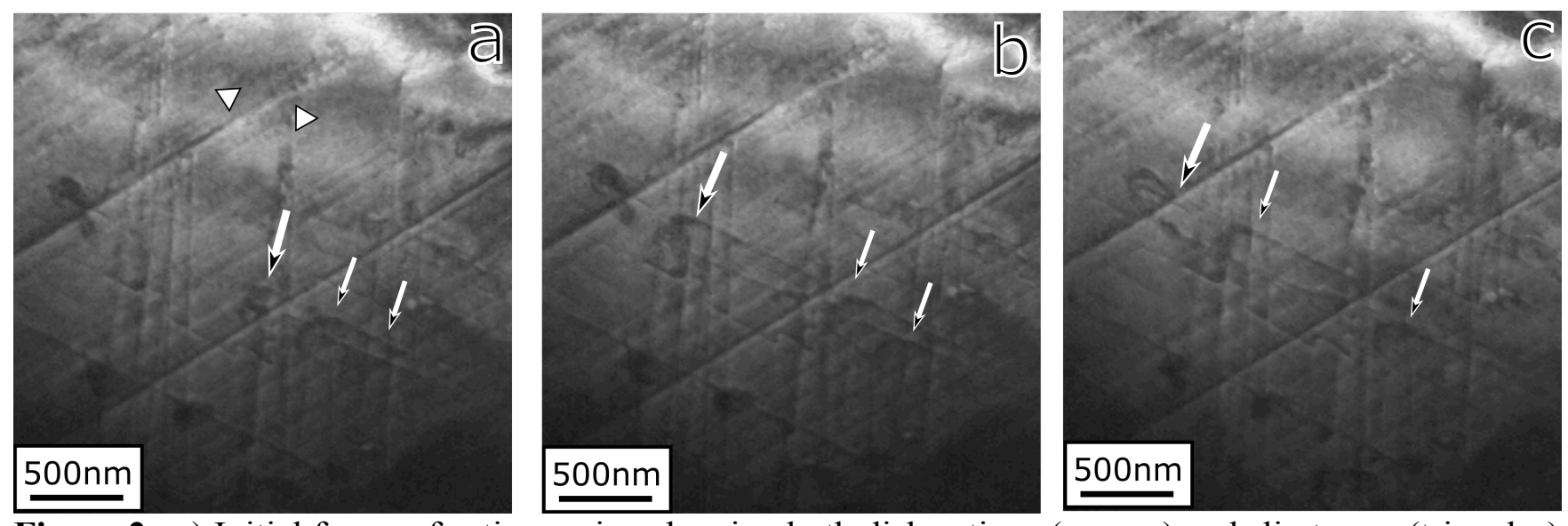

Figure 2. a) Initial frame of active region showing both dislocations (arrows) and slip traces (triangles). b-c) Three dislocations moving in a "jerky" motion past slip traces, with the lead dislocation encountering another dislocation annihilating. 